\title{
Magnetic Resonance Elastography and Diffusion Weighted Imaging in the Evaluation of Hepatic Fibrosis in Chronic Hepatitis B
}

Tiffany P. Hennedige ${ }^{1}$, Gang Wang ${ }^{1,2}$, Fiona P. Leung ${ }^{1,3}$, Hind S. Alsaif ${ }^{1,4}$, Lynette LS Teo ${ }^{1}$, Seng Gee Lim ${ }^{5}$, Aileen Wee ${ }^{6}$, and Sudhakar K. Venkatesh ${ }^{7}$

${ }^{\prime}$ Department of Diagnostic Imaging, National University of Singapore, Singapore, ${ }^{2}$ Department of Biomedical Engineering, University of Calgary, Calgary, Canada, ${ }^{3}$ Department of Radiology, King Fahd Hospital of the University, Dammam University College of Medicine, Al Khobar, Saudi Arabia, ${ }^{4}$ South West Radiology, Liverpool, Australia, Departments of ${ }^{5}$ Gastroenterology and Hepatology, ${ }^{6}$ Pathology, National University of Singapore, Singapore, and ${ }^{7}$ Department of Radiology, Mayo Clinic, Rochester, MN, USA

See editorial on page 321.

Background/Aims: Comparison of the accuracy of magnetic resonance elastography (MRE) and diffusion weighted imaging (DWI) for the diagnosis of liver fibrosis in patients with chronic hepatitis $\mathrm{B}(\mathrm{CHB})$. Methods: In this retrospective analysis, we investigated 63 patients with $\mathrm{CHB}$ and liver fibrosis. DWI was performed with both breath-hold (DWI$\mathrm{BH})$ and free-breathing (DWI-FB) sequences $(b=0,500)$. The mean liver stiffness and apparent diffusion coefficient (ADC) were calculated by drawing regions of interest maps. Fibrosis staging according to the METAVIR system was independently performed by an experienced pathologist. A receiver operating curve (ROC) analysis was conducted to determine the accuracy of MRE, DWI-BH and DWI-FB in the detection and stratification of liver fibrosis. The performance of the detection of significant fibrosis ( $\geq F 2$ ), advanced fibrosis ( $\geq F 3)$, and cirrhosis (F4) was also evaluated by comparing areas under the ROC. Results: There was a moderate and significantly negative correlation between the ADC values and liver stiffness. The accuracies for the detection of $\geq F 2 / \geq F 3 / F 4$ stage fibrosis with DWI-FB, DWI-BH and MRE were 0.84/0.76/0.72, $0.72 / 0.83 / 0.79$ and 0.99/0.99/0.98, respectively. The performance of MRE was significantly better than DWI-FB and DWI-BH. There were no significant differences between the performance of DWI-FB and DWI-BH. Conclusions: MRE is more accurate than DWI for the detection and stratification of liver fibrosis in CHB. (Gut Liver 2017;11:401-408)

Key Words: Elastography; Liver cirrhosis; Diffusion weighted imaging; Accuracy; Cirrhosis

\section{INTRODUCTION}

Chronic liver disease results from many different etiologies and the final end result is scarring of the liver parenchyma leading to cirrhosis and its associated complications. Treatment of the etiology of chronic liver disease especially in the early stages of fibrosis may result in reversal of fibrosis. ${ }^{1-4}$ Traditionally, liver biopsy has been the reference standard for detection and staging of liver fibrosis but is limited by its invasive nature, sampling error, interobserver variability and reluctance of patients to undergo repeated biopsies for monitoring treatment response $^{5-9}$ Accurate noninvasive tests are therefore required for detection and staging of fibrosis. Imaging tests, and in particular magnetic resonance imaging (MRI) has been the focus of investigation in this direction as it is safer compared to computed tomography for repeated use. Morphological features of advanced liver fibrosis are easily appreciated on conventional MRI sequences, but are not sensitive or specific to detect early stages of fibrosis. ${ }^{10}$ Detection of early fibrosis is an unmet need as newer drugs are now available that can potentially reverse fibrosis. Quantitative MRI techniques that can measure degree of fibrosis may be useful. Two MRI based techniques namely, diffusion weighted imaging (DWI) and magnetic resonance elastography (MRE) are particularly of interest as both techniques do not require intravenous contrast medium and are easily repeatable.

DWI is a sensitive MRI technique. Diffusion of free water causes decrease in liver signal due to motion and is quantified as apparent diffusion coefficient (ADC). Liver fibrosis by

Correspondence to: Sudhakar K. Venkatesh

Diagnostic Radiology, Mayo Clinic, 200 First Street SW, Rochester 55902, MN, USA

Tel: +1-507284-1728, Fax: +1-507-284-2405, E-mail: venkatesh.sudhakar@mayo.edu

Received on February 11, 2016. Revised on June 5, 2016. Accepted on June 30, 2016. Published online December 16, 2016

pISSN 1976-2283 eISSN 2005-1212 https://doi.org/10.5009/gnl16079

(a) This is an Open Access article distributed under the terms of the Creative Commons Attribution Non-Commercial License (http://creativecommons.org/licenses/by-nc/4.0) which permits unrestricted non-commercial use, distribution, and reproduction in any medium, provided the original work is properly cited. 
increasing barriers and associated reduction in vascularity leads to reduced ADC values. Studies have demonstrated that DWI is an accurate technique for detection of fibrosis ${ }^{11,12}$ while other studies have shown poor performance of DWI. ${ }^{13,14}$ However, there is no established standard technique for performing DWI for staging liver fibrosis and optimal b-values remain unknown. MRE measures the stiffness of tissues under investigation by propagating mechanical shear waves into the tissues and deriving stiffness values with inversion of the wave information. MRE has been established as an accurate method in the evaluation of chronic liver disease and the liver stiffness is considered an accurate marker for staging of liver fibrosis in chronic viral hepatitis. $^{15,16}$

One earlier study has also compared DWI and MRE in a study population comprised of liver fibrosis from various etiologies. ${ }^{17}$ The degree and pattern of fibrosis are variable with different etiologies of chronic liver disease. It is therefore desirable to have studies comparing the techniques in one particular etiology and eliminating the possible contamination of the data due to other etiologies. Hence, we studied cases of chronic hepatitis B (CHB) that underwent liver biopsy for confirmation of liver fibrosis.

\section{MATERIALS AND METHODS}

\section{Study population}

A retrospective search for patients with $\mathrm{CHB}$ who underwent a liver MRI study and a liver biopsy within 6 months was made. The inclusion criteria were (1) a liver biopsy performed within 6 months of MRI and (2) MRI study included MRE and DWI. We excluded cases with biopsy performed $>6$ months from MRE to minimize the effect on the study due to possible progression of disease. Liver biopsy was performed in these patients to stage liver fibrosis for management purposes (initiating antiviral therapy or follow-up). Liver biopsy sample was considered adequate when there were $\geq 10$ portal tracts for accurate staging of liver fibrosis. Seventy-five subjects between March 2009 and December 2012 were eligible for inclusion. Eight patients were excluded as they received treatment between the MRI study and liver biopsy and four patients were excluded as liver biopsy sample was considered inadequate. The final study group consisted of 63 clinical subjects with 44 males and 19 females.

\section{MRI examination}

MRI was performed on a 1.5-T clinical scanner (Signa HDx TwinSpeed; GE Healthcare, Waukesha, WI, USA) using a 16-channel surface coil as receiver. All subjects were prepared with 4 to 6 hours fasting as per clinical liver MRI protocol. Plain water was allowed to maintain hydration. MRE and DWI sequences were performed along the routine liver MRI protocol. MRE was performed with a modified GRE sequence with the following parameters: repetition time (TR)/echo time (TE), 50/24 msec; flip angle 30 , bandwidth $31.25 \mathrm{kHz}$, matrix of $256 \times 128$; asymmetric 75\% field of view. Four slices of 10-mm thickness was obtained through the largest cross-section of the liver with four breath hold (in expiration) duration of 16 to 22 seconds each depending on the size of the patient. The entire sequence took less than 2 to 3 minutes to perform.

Echo planar imaging based DWI was performed with free breathing (DWI-FB) technique using b-values 0 and 500; TR/ $\mathrm{TE}=3,000-5,000 / 91 \mathrm{~ms}$; matrix size $128 \times 256$; slice thickness of $5 \mathrm{~mm}$ with $2 \mathrm{~mm}$ interslice gap; bandwidth $1.5 \mathrm{kHz}, \mathrm{NEX}=5$, parallel imaging factor of 2 and gradients sensitized in all three directions. The entire liver was covered with this sequence and the total acquisition time was 4 to 6 minutes.

The second DWI sequence was performed in breath hold (DWI-BH) with b-value of 500 sensitized in all three directions; $\mathrm{TR} / \mathrm{TE}=3,000-4,000 / 76 \mathrm{~ms}$; matrix size $128 \times 256$; slice thickness of 7-mm; NEX=1, parallel imaging factor of 2. Four slices were obtained corresponding to the levels of MRE sections. The breath hold was performed in expiration to correspond to the MRE sequence. The entire sequences took less than 1 to 2 minutes to perform.

The MRE data was automatically processed and stiffness maps were generated. ADC maps were generated on the workstation (Advantage Windows 4.2; GE Healthcare).

\section{Quantification of MRE and DWI data}

The stiffness maps from MRE and the ADC maps from DWI were viewed simultaneously on the work station (Advantage Windows 4.0; GE Healthcare). The four slices of MRE and DWI$\mathrm{BH}$ were easily matched. In the case of ADC maps from the DWI-FB, the slices were matched to the closest possible using slice position with vessels and fissures as landmarks. Two readers in consensus; one experienced reader (abdominal radiologist with 10 years' experience with DWI and 5 years' experience with MRE) and the second reader with 1 year of abdominal fellowship experience. Both readers blinded to histology results placed regions of interest (ROIs) in the right lobe of the liver on each slice of the MRE obtained and copied them onto ADC maps at the corresponding levels in both DWI-BH and DWIFB. Two or more ROIs were drawn on the right lobe of the liver carefully avoiding the liver edge, vessels, fissures and areas of motion artifacts on the magnitude images of the MRE as well as DWI. Care was also taken to avoid areas of wave interference seen on the MRE wave images and any other artifacts on the DWI images. Each ROI placed was at least $100 \mathrm{~mm}^{2}$ in area in the right lobe. The left lobe was avoided as the cardiac pulsations may introduce artifacts and unreliable ADC and stiffness values. The mean and standard deviations (SD) of the ROIs in each slice were recorded and mean of the readings from ROIs placed in all four slices were taken to represent the mean stiffness value kilopascals $(\mathrm{kPa})$ and mean $\operatorname{ADC}\left(10^{-3} / \mathrm{mm}^{2}\right)$ of the liver tissue in that subject. Examples of representative $\mathrm{ADC}$ and stiffness maps along with corresponding T2W and DWI images 
T2-W
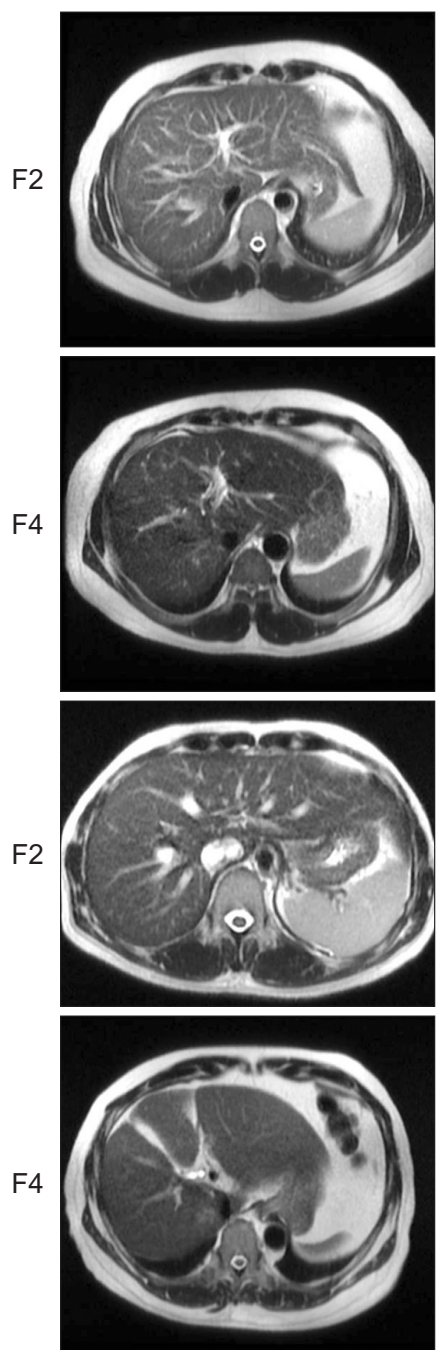

DWI
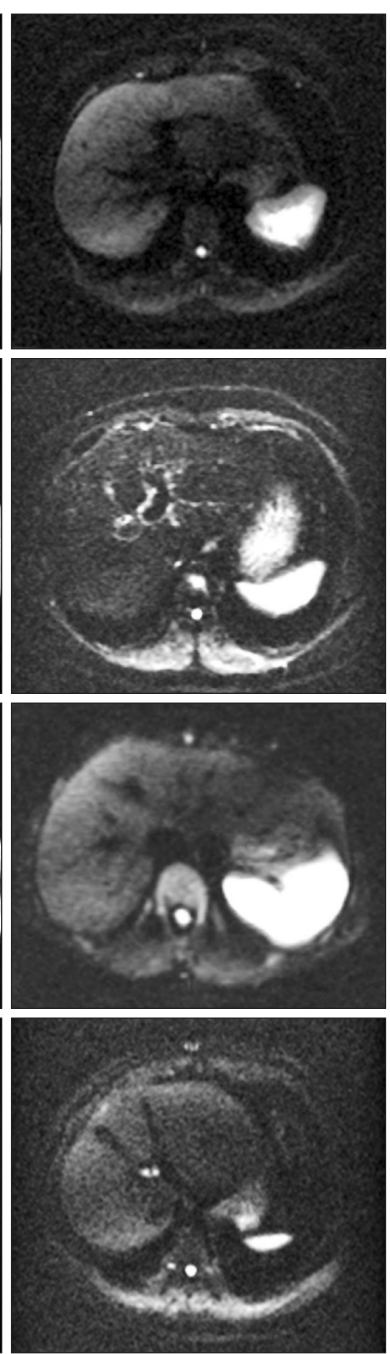

ADC
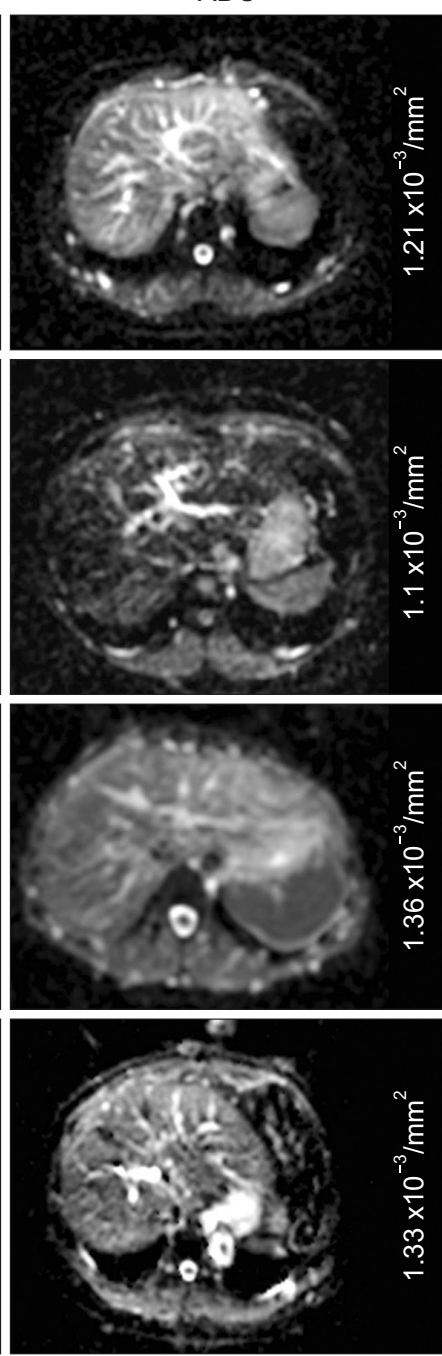

MRE
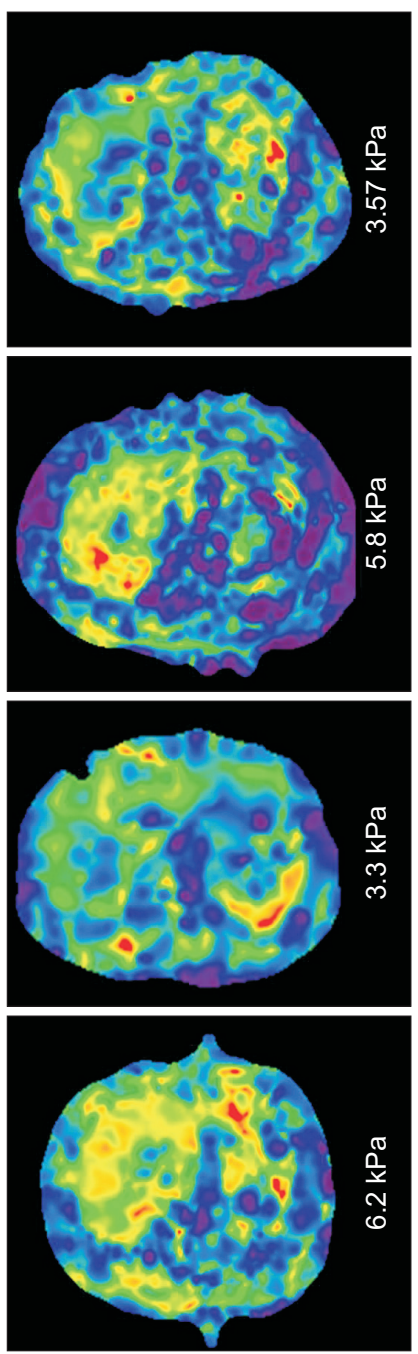

Fig. 1. Examples of representative axial T2 weighted images (first column), diffusion weighted imaging (DWI; second column), apparent diffusion coefficient (ADC) maps (third column) and stiffness maps from magnetic resonance elastography (MRE; fourth column). DWI images in the top and third rows were obtained from breath-hold DWI sequences, whereas the second and fourth rows were obtained from free-breathing DWI sequences. In the top and second rows, the ADC and MRE values correctly classified the cases as stage F2 and F4 fibrosis. In the third and fourth rows, the MRE values correctly classified the cases as stage F2 and F4 fibrosis; however, the ADC values failed to correctly classify these cases.

are demonstrated in Fig. 1.

One experienced hepatopathologist with special interest in liver pathology (A.W.) independently interpreted liver biopsy samples and resection samples for staging of liver fibrosis. The liver fibrosis was staged according to the METAVIR score $:^{18}$ F0=no fibrosis; F1=periportal fibrosis; F2=periportal fibrosis with few septae; F3=septal fibrosis; and F4=cirrhosis. The necroinflammatory activity was also scored as A1=mild, $\mathrm{A} 2=$ moderate, and A3=severe. The 20 healthy volunteers did not undergo any liver biopsy and were presumed to have normal liver parenchyma and no fibrosis.

\section{Statistical analysis}

The mean \pm SD of the ADC and liver stiffness values were calculated for the clinical subjects with fibrosis stages F0 through
F4 and the normal volunteers. Mean ADC values were expressed as $10^{-3} / \mathrm{mm}^{2}$ and liver stiffness as $\mathrm{kPa}$. Correlation between mean ADC and stiffness values were tested with Pearson correlation coefficient analysis. Kruskal-Wallis test was used to compare the $\mathrm{ADC}$ and stiffness values between different fibrosis stages and inflammation grades. For the statistical purposes we combined F0 and F1 stages as one group F0-1 stage. Receiver operating curve analysis was performed for prediction of significant fibrosis (F0-1 vs F2-4; $\geq$ F2), severe fibrosis (F0-2 vs F3$4 ; \geq \mathrm{F} 3$ ) and cirrhosis (F0-3 vs F4; F4). Likelihood ratio (LR), sensitivity, specificity, positive predictive value, negative predictive value and accuracy for best cutoff value determined by the software were reported for ADC and liver stiffness measurements. Comparison accuracies were performed by comparing areas under ROC (AUROC) using the method described by DeLong et 
al. ${ }^{19}$ Statistical analyses were performed using a commercially available MedCalc Statistical Software version 12.7.7 (MedCalc Software bvba, Ostend, Belgium).

\section{RESULTS}

\section{Patient data}

The study group comprised of 63 subjects with 44 males and 19 females. The mean \pm SD age was $50.1 \pm 12$ years (range, 23 to 72 years) and mean \pm SD body mass index was $24.9 \pm 4.0 \mathrm{~kg} / \mathrm{m}^{2}$ (95\% CI, 23.8 to $25.8 \mathrm{~kg} / \mathrm{m}^{2}$ ). The mean duration between MRE and biopsy was 62.6 days (95\% CI, 18.3 to 22.8 days).

\section{Liver biopsy}

The mean cumulative liver biopsy length was $20.6 \mathrm{~mm}$ (95\% CI, 18.3 to $22.9 \mathrm{~mm}$ ). The METAVIR stages of fibrosis were F0, 12 patients; F1, 12 patients; F2, 10 patients; F3, eight patients; and F4, 21 patients. The inflammation grades were A0 in 13, A1 in 41 , and $\mathrm{A} 2$ in nine patients.

\section{Diffusion weighted imaging}

The mean \pm SD of $A D C$ of the study group was $125.9 \pm 24.5$

Table 1. Apparent Diffusion Coefficient and Magnetic Resonance Elastography for Different Stages of Fibrosis (n=63)

\begin{tabular}{ccccc}
\hline Fibrosis stage & No. & $\begin{array}{c}\text { DWI-BH ADC, } \\
10^{-3} / \mathrm{mm}^{2}\end{array}$ & $\begin{array}{c}\text { DWI-FB ADC, } \\
10^{-3} / \mathrm{mm}^{2}\end{array}$ & $\begin{array}{c}\text { MRE stiffness, } \\
\mathrm{kPa}\end{array}$ \\
\hline F0 & 12 & $136.4 \pm 26.2$ & $150.4 \pm 19.3$ & $2.6 \pm 0.3$ \\
F1 & 12 & $139.2 \pm 17.7$ & $140.6 \pm 30.7$ & $2.8 \pm 0.2$ \\
F2 & 10 & $140.0 \pm 12.9$ & $116.7 \pm 23.8$ & $3.5 \pm 0.3$ \\
F3 & 8 & $110.2 \pm 33.7$ & $114.0 \pm 23.6$ & $4.3 \pm 0.7$ \\
F4 & 21 & $111.6 \pm 15.8$ & $111.7 \pm 12.9$ & $6.5 \pm 2.1$ \\
\hline
\end{tabular}

Data are presented as mean \pm SD.

DWI-BH, diffusion weighted imaging-breath hold; ADC, apparent diffusion coefficient; DWI-FB, diffusion weighted imaging-free breathing; MRE, magnetic resonance elastography.

Table 2. Apparent Diffusion Coefficient and Magnetic Resonance Elastography for Different Grades of Inflammation ( $\mathrm{n}=63)$

\begin{tabular}{ccccc}
\hline Inflammation grade & No. & $\begin{array}{c}\text { DWI-BH ADC, } \\
10^{-3} / \mathrm{mm}^{2}\end{array}$ & $\begin{array}{c}\text { DWI-FB ADC, } \\
10^{-3} / \mathrm{mm}^{2}\end{array}$ & $\begin{array}{c}\text { MRE stiffness, } \\
\mathrm{kPa}\end{array}$ \\
\hline A0 & 13 & $123.8 \pm 26.4$ & $131.4 \pm 28.7$ & $4.3 \pm 1.7$ \\
A1 & 41 & $127.9 \pm 21.4$ & $123.4 \pm 27.2$ & $4.1 \pm 2.0$ \\
A2 & 9 & $119.9 \pm 35.0$ & $127.7 \pm 18.5$ & $5.5 \pm 2.4$ \\
\hline
\end{tabular}

Data are presented as mean \pm SD.

DWI-BH, diffusion weighted imaging-breath hold; ADC, apparent diffusion coefficient; DWI-FB, diffusion weighted imaging-free breathing; MRE, magnetic resonance elastography.

A

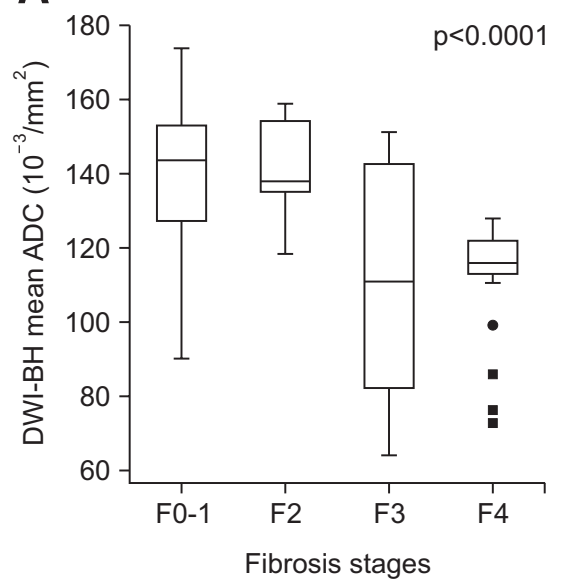

B

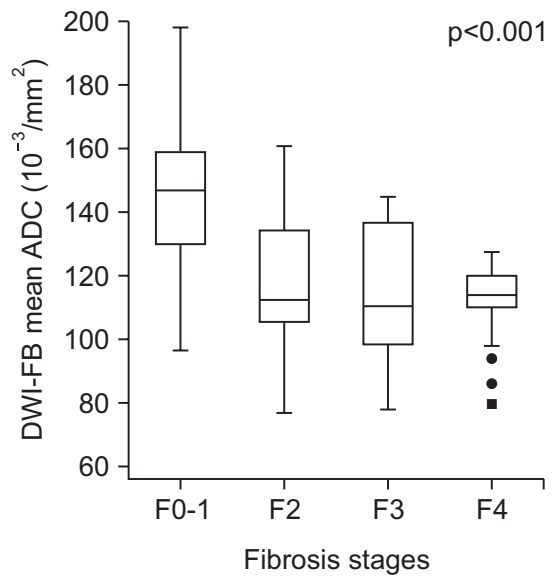

C

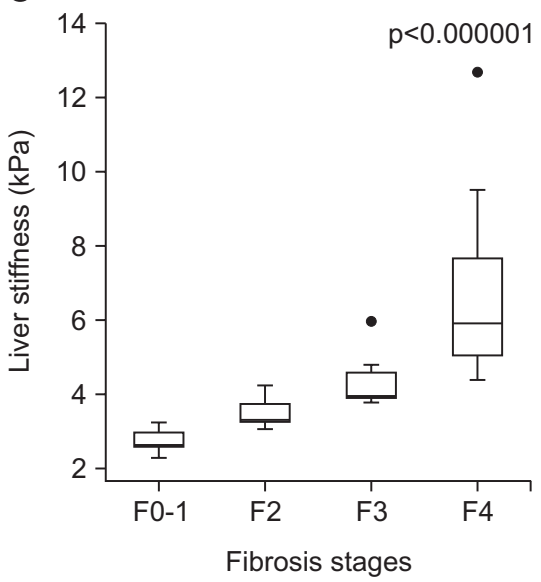

Fig. 2. Box and whisker plots demonstrate the apparent diffusion coefficient (ADC) values for diffusion weighted imaging-breath hold (A) and diffusion weighted imaging-free breathing (B) and the liver stiffness (C) values for magnetic resonance elastography (MRE) in liver stages F0-1 through F4. 


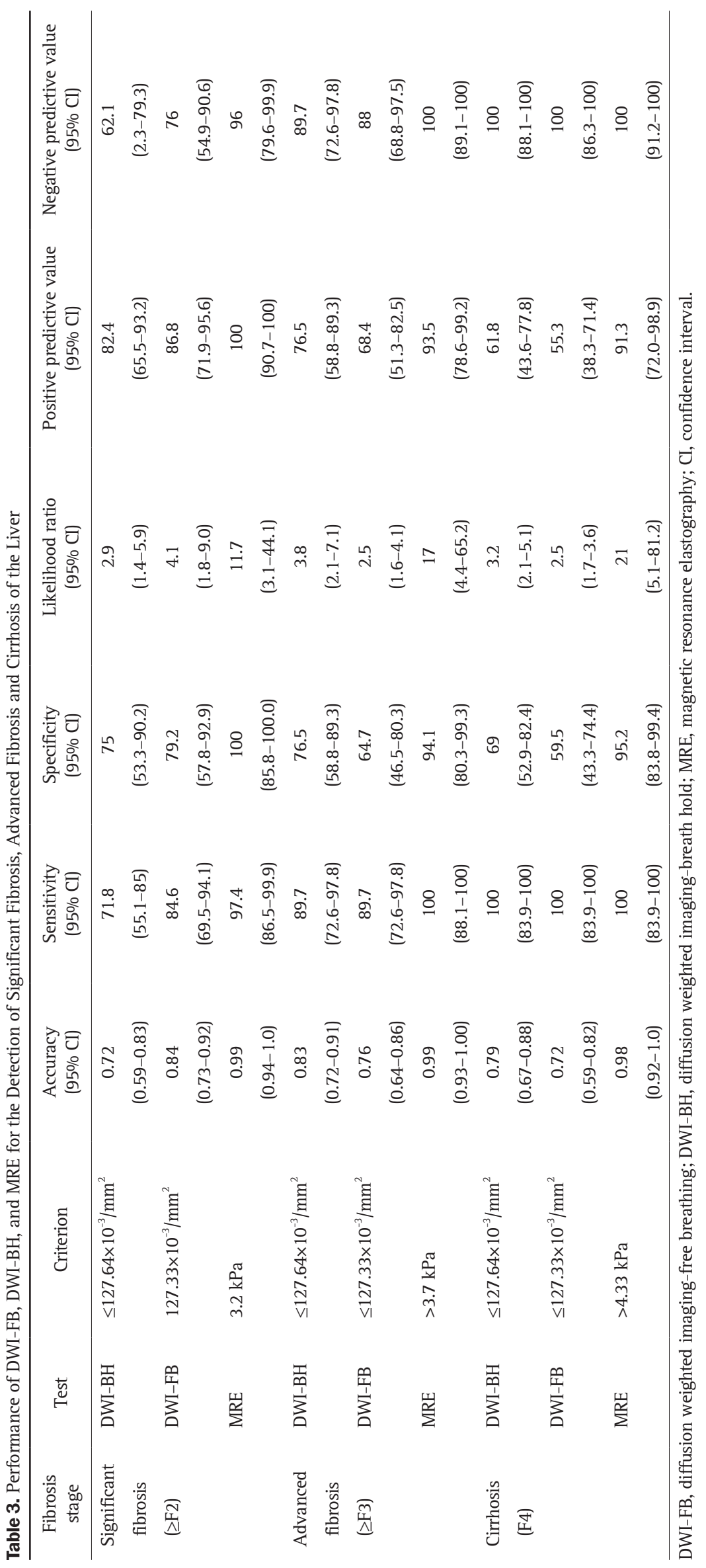


(95\% CI, 119.8 to 132.1 ; range, 63.6 to 173.7 ) for DWI-BH and $125.7 \pm 26.3$ (95\% CI, 119.1 to 132.3 ; range, 76.7 to 198.1 ) for DWI-FB (Table 1). Significant negative correlation existed between fibrosis stages and mean ADC values on both DWI-BH $(\mathrm{r}=-0.49, \mathrm{p}<0.0001)$ and DWI-FB $(\mathrm{r}=-0.56, \mathrm{p}<0.0001)$. The mean $\mathrm{ADC}$ values of F0 to F1 and F2 stages were significantly lower than F3 and F4 $(\mathrm{p}<0.05)$ stages on the DWI-BH sequence (Fig. 2A). On the DWI-FB sequence only F0-1 stages mean ADC was significantly lower than all other stages (Fig. 2B). There were no significant differences in mean $\mathrm{ADC}$ values among inflammation grades A0 through A2 (Table 2). Receiver operating curve analysis showed moderate to good accuracy, sensitivity and specificity of both DWI-BH and DWI-FB for detection significant fibrosis advanced fibrosis and cirrhosis (Table 3). The LRs with DWI-BH and DWI-FB for significant fibrosis, advanced fibrosis and cirrhosis were 2.9, 3.8 and 3.2; and 4.1, 2.5 and 2.5, respectively.

\section{Magnetic resonance elastography}

The mean \pm SD of the liver stiffness was $3.8 \pm 2.1 \mathrm{kPa}(95 \% \mathrm{CI}$, 3.3 to $4.2 \mathrm{kPa}$; range, 1.7 to $12.7 \mathrm{kPa}$ ). There was significant positive correlation between liver stiffness and fibrosis stages $(r=0.93, p<0.0001)$. Trend of increasing stiffness with fibrosis stages was observed.

The mean liver stiffness of stages F0-1 through F4 were significantly different from each other (Fig. 2C). There were no significant differences in the mean stiffness of liver between different inflammation grades. The positive correlation between MRE and fibrosis stages remained significant after correcting for inflammation grade $(r=0.76, p<0.001)$. The $L R$ for significant fibrosis, advanced fibrosis and cirrhosis were 11.7, 17 and 21, respectively.

\section{MRE versus DWI}

Pearson correlation coefficient analysis showed moderate correlation between mean ADC measured with DWI-BH and DWIFB. There was also moderate negative but significant correlation between MRE and ADC with DWI-BH and DWI-FB (Table 4). Comparison of AUROCs of MRE, DWI-FB and DWI-BH showed significantly better performance of MRE for significant fibrosis (0.99 vs 0.72 and 0.85), advanced fibrosis (0.98 vs 0.79 and 0.72 ) and cirrhosis (0.99 vs 0.83 and 0.76) (Fig. 3). There were no significant differences between DWI-FB and DWI-BH.

Table 4. Correlation between Apparent Diffusion Coefficient Values and Stiffness Measurements

\begin{tabular}{lccc}
\hline & DWI-BH mean ADC & DWI-FB mean ADC & MRE \\
\hline DWI-BH mean ADC & - & $r=0.256, p=0.04$ & $r=-0.466, p=0.0001$ \\
DWI-FB mean ADC & $r=0.256, p=0.043$ & - & $r=-0.410, p=0.0009$ \\
\hline
\end{tabular}

DWI-BH, diffusion weighted imaging-breath hold; ADC, apparent diffusion coefficient; DWI-FB, diffusion weighted imaging-free breathing; MRE, magnetic resonance elastography.

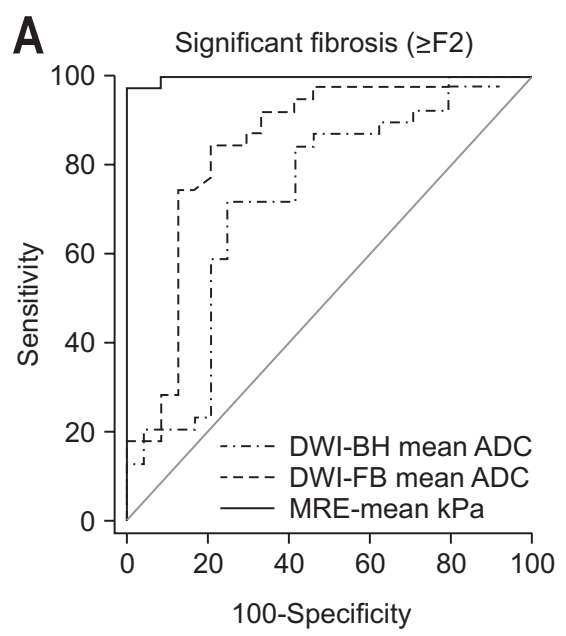

MRE vs DWI-BH, $p<0.0001$ MRE vs DWI-FB, $p<0.008$ DWI-BH vs DWI-FB, $p=0.18$

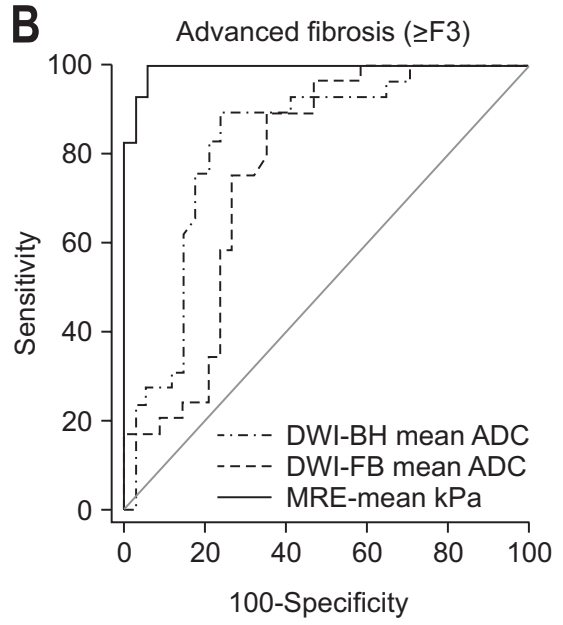

MRE vs DWI-BH, $\mathrm{p}=0.0005$ MRE vs DWI-FB, $\mathrm{p}<0.0001$ DWI-BH vs DWI-FB, $p=0.35$

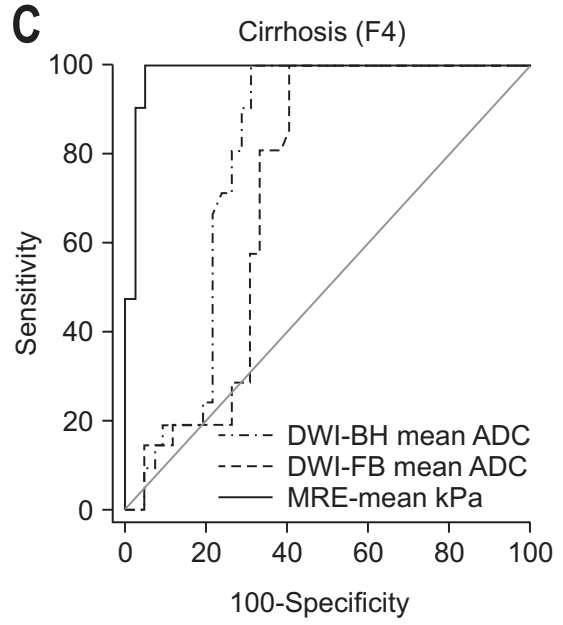

MRE vs DWI-BH, $p=0.0026$ MRE vs DWI-FB, $p=0.0002$ DWI-BH vs DWI-FB, $p=0.42$

Fig. 3. Comparison of the area under receiver operating curves indicated significantly better performance of magnetic resonance elastography (MRE) for all stages of fibrosis than that of diffusion weighted imaging-breath hold (DWI-BH) and diffusion weighted imaging-free breathing (DWI-FB). $\mathrm{ADC}$, apparent diffusion coefficient. 


\section{DISCUSSION}

Our study results show that MRE is more accurate than DWI for evaluation of liver fibrosis in CHB. MRE performed significantly better than both DWI-BH and DWI-FB sequences. DWI although showed a significant correlation with fibrosis stages and moderate to good performance in differentiating significant fibrosis, advanced fibrosis and cirrhosis could not match MRE accuracy. The lower performance of DWI is probably related to confounding factors such as perfusion and inflammation which can affect the ADC values. Also in our study we showed that different grades of inflammation did not influence mean ADC values and liver stiffness. Further after correcting for grades of inflammation MRE still showed excellent correlation with fibrosis stages.

Wang et al. ${ }^{17}$ performed a similar study comparing MRE with diffusion weighted MRI. In their study MRE had greater predictive ability in distinguishing the stages of liver fibrosis than DWI. In their study they used three b-values of 50, 500, and 1,000 $\mathrm{s} / \mathrm{mm}^{2}$ and the MRE technique was similar to ours. The authors demonstrated higher accuracy and sensitivity and specificity similar to our study. In a meta-analysis study comparing DWI with MRE, Wang et al. ${ }^{20}$ studied 14 studies with 9 on DWI, 4 on MRE and 1 on DWI and MRE and concluded that MRE is more reliable for staging liver fibrosis as compared with DWI with a high combination of sensitivity, specificity, LRs, diagnostic odds ratio and area under curve. On a separate note, Zou et al. ${ }^{21}$ performed a comparison study in an animal model and showed that AUC of MRE was significantly larger than DWI for predicting all stages of hepatic fibrosis.

In another study by Kim et al. ${ }^{22}$ for assessment of advanced fibrosis and cirrhosis, DWI with b-values of 50, 400, and 800 $\mathrm{s} / \mathrm{mm}^{2}$ performed inferior to transient elastography measured liver stiffness.

Some limitations are noted in this study; it is a retrospective study, there was no evaluation on the possible influence of steatosis and iron overload on ADC measurements. It has been shown that fatty change and iron overload may affect ADC measurements. We did not assess interobserver variability between readers for ADC and MRE as they were performed in consensus. However, it has been shown previously that interobserver correlation is excellent for $\mathrm{MRE}^{10}$ and moderate for ADC measurements. ${ }^{21}$ We used b-values of 500 , which is the midrange and most suitable for abdominal imaging. However, the choice of b-values is variable across institutions and publications. Therefore, the ADC values may not be comparable across various platforms; however, our objective was to compare the diagnostic performance of MRE and DWI. In the study by Wang et al. ${ }^{17}$, they used b-values of 50, 500, and 1,000, but DWI performed inferior to MRE.

We used histology as reference standard which has limitations of sampling error; however, we minimized this with inter- pretation by an experienced pathologist with specialization in liver pathology.

In conclusion, it is of importance to develop a noninvasive method which is able to stratify the varying stages of fibrosis accurately, particularly in early fibrosis where the disease is potentially reversible. Our results show that MRE is better able to perform this function relative to DWI with MRE demonstrated higher accuracy, sensitivity and specificity for predicting all stages of fibrosis compared with DWI. This is consistent with findings from other studies and lends further support to increasing use of MRE with the potential to replacement of liver biopsy as a gold standard.

\section{CONFLICTS OF INTEREST}

No potential conflict of interest relevant to this article was reported.

\section{REFERENCES}

1. Friedman SL, Bansal MB. Reversal of hepatic fibrosis: fact or fantasy? Hepatology 2006;43(2 Suppl 1):S82-S88.

2. Enomoto M, Mori M, Ogawa T, et al. Usefulness of transient elastography for assessment of liver fibrosis in chronic hepatitis B: regression of liver stiffness during entecavir therapy. Hepatol Res 2010;40:853-861.

3. Bortolotti F, Guido M, Cadrobbi P, et al. Spontaneous regression of hepatitis B virus-associated cirrhosis developed in childhood. Dig Liver Dis 2005;37:964-967.

4. Bortolotti F, Iorio R, Nebbia G, et al. Interferon treatment in children with chronic hepatitis C: long-lasting remission in responders, and risk for disease progression in non-responders. Dig Liver Dis 2005;37:336-341.

5. Colloredo G, Guido M, Sonzogni A, Leandro G. Impact of liver biopsy size on histological evaluation of chronic viral hepatitis: the smaller the sample, the milder the disease. J Hepatol 2003;39:239244

6. Guido M, Rugge M. Liver biopsy sampling in chronic viral hepatitis. Semin Liver Dis 2004;24:89-97.

7. Regev A, Berho M, Jeffers LJ, et al. Sampling error and intraobserver variation in liver biopsy in patients with chronic HCV infection. Am J Gastroenterol 2002;97:2614-2618.

8. Westin J, Lagging LM, Wejstål R, Norkrans G, Dhillon AP. Interobserver study of liver histopathology using the Ishak score in patients with chronic hepatitis C virus infection. Liver 1999;19:183187.

9. Cadranel JF, Rufat P, Degos F. Practices of liver biopsy in France: results of a prospective nationwide survey. For the Group of Epidemiology of the French Association for the Study of the Liver (AFEF). Hepatology 2000;32:477-481.

10. Venkatesh SK, Yin M, Takahashi N, Glockner JF, Talwalkar JA, Ehman RL. Non-invasive detection of liver fibrosis: MR imaging 
features vs. MR elastography. Abdom Imaging 2015;40:766-775.

11. Ayvaz S, Tuncel SA, Can G, et al. Efficacy of diffusion-weighted mri in the evaluation of extrahepatic cholestasis-related hepatic fibrosis. Turk J Med Sci 2015;45:686-693.

12. Hu XR, Cui XN, Hu QT, Chen J. Value of MR diffusion imaging in hepatic fibrosis and its correlations with serum indices. World $\mathrm{J}$ Gastroenterol 2014;20:7964-7970.

13. Ding Y, Rao SX, Zhu T, Chen CZ, Li RC, Zeng MS. Liver fibrosis staging using T1 mapping on gadoxetic acid-enhanced MRI compared with DW imaging. Clin Radiol 2015;70:1096-1103.

14. Park HS, Kim YJ, Yu MH, Choe WH, Jung SI, Jeon HJ. ThreeTesla magnetic resonance elastography for hepatic fibrosis: comparison with diffusion-weighted imaging and gadoxetic acidenhanced magnetic resonance imaging. World J Gastroenterol 2014;20:17558-17567.

15. Venkatesh SK, Wang G, Lim SG, Wee A. Magnetic resonance elastography for the detection and staging of liver fibrosis in chronic hepatitis B. Eur Radiol 2014;24:70-78.

16. Ichikawa S, Motosugi U, Ichikawa T, et al. Magnetic resonance elastography for staging liver fibrosis in chronic hepatitis C. Magn Reson Med Sci 2012;11:291-297.
17. Wang Y, Ganger DR, Levitsky J, et al. Assessment of chronic hepatitis and fibrosis: comparison of MR elastography and diffusionweighted imaging. AJR Am J Roentgenol 2011;196:553-561.

18. Bedossa P, Poynard T. An algorithm for the grading of activity in chronic hepatitis C: the METAVIR Cooperative Study Group. Hepatology 1996;24:289-293.

19. DeLong ER, DeLong DM, Clarke-Pearson DL. Comparing the areas under two or more correlated receiver operating characteristic curves: a nonparametric approach. Biometrics 1988;44:837-845.

20. Wang QB, Zhu H, Liu HL, Zhang B. Performance of magnetic resonance elastography and diffusion-weighted imaging for the staging of hepatic fibrosis: a meta-analysis. Hepatology 2012;56:239247.

21. Zou LQ, Chen J, Pan L, Jiang JZ, Xing W. Comparison of magnetic resonance elastography and diffusion-weighted imaging for staging hepatic fibrosis. Chin Med J (Engl) 2015;128:620-625.

22. Kim SU, Kim YC, Choi JS, et al. Can preoperative diffusionweighted MRI predict postoperative hepatic insufficiency after $\mathrm{cu}-$ rative resection of HBV-related hepatocellular carcinoma? A pilot study. Magn Reson Imaging 2010;28:802-811. 